NBER WORKING PAPER SERIES

\title{
WHAT WILL BE THE ECONOMIC IMPACT OF COVID-19 IN THE US? ROUGH ESTIMATES OF DISEASE SCENARIOS
}

\author{
Andrew Atkeson \\ Working Paper 26867 \\ http://www.nber.org/papers/w26867 \\ NATIONAL BUREAU OF ECONOMIC RESEARCH \\ 1050 Massachusetts Avenue \\ Cambridge, MA 02138 \\ March 2020
}

All errors are mine. The views expressed here are entirely my own and not official statements of the Federal Reserve Bank of Minneapolis, the Federal Reserve, or the National Bureau of Economic Research.

NBER working papers are circulated for discussion and comment purposes. They have not been peer-reviewed or been subject to the review by the NBER Board of Directors that accompanies official NBER publications.

(C) 2020 by Andrew Atkeson. All rights reserved. Short sections of text, not to exceed two paragraphs, may be quoted without explicit permission provided that full credit, including () notice, is given to the source. 
What Will Be the Economic Impact of COVID-19 in the US? Rough Estimates of Disease

Scenarios

Andrew Atkeson

NBER Working Paper No. 26867

March 2020

JEL No. C0,E0

\begin{abstract}
This note is intended to introduce economists to a simple SIR model of the progression of COVID-19 in the United States over the next 12-18 months. An SIR model is a Markov model of the spread of an epidemic in a population in which the total population is divided into categories of being susceptible to the disease (S), actively infected with the disease (I), and recovered (or dead) and no longer contagious (R). How an epidemic plays out over time is determined by the transition rates between these three states. This model allows for quantitative statements regarding the tradeoff between the severity and timing of suppression of the disease through social distancing and the progression of the disease in the population. Example applications of the model are provided. Special attention is given to the question of if and when the fraction of active infections in the population exceeds $1 \%$ (at which point the health system is forecast to be severely challenged) and $10 \%$ (which may result in severe staffing shortages for key financial and economic infrastructure) as well as the cumulative burden of the disease over an 18 month horizon.
\end{abstract}

Andrew Atkeson

Bunche Hall 9381

Department of Economics

UCLA

Box 951477

Los Angeles, CA 90095-1477

and NBER

andy@atkeson.net 


\section{Introduction}

In the face of the rapidly growing COVID-19 pandemic, a wide variety of SIR models of the progression of this epidemic are being used by public health experts to generate scenarios that are being used to guide decisions to recommend and impose increasingly severe mitigation measures on economies worldwide. ${ }^{1}$ Economists are not fully familiar with the quantitative implications of these models and thus are not fully engaged in the policy discussion regarding the tradeoff between the public health and economic implications of these mitigation and social distancing measures. ${ }^{2}$

This note is intended to introduce economists to a simple SIR model of the progression of COVID-19 in the United States over the next 12-18 months. An SIR model is a Markov model of the spread of an epidemic in a population in which the total population is divided into categories of being susceptible to the disease $(\mathrm{S})$, actively infected with the disease (I), and recovered (or dead) and no longer contagious $(\mathrm{R}){ }^{3}{ }^{3}$ How an epidemic plays out over time is determined by the transition rates between these three states. These transition rates are determined by characteristics of the underlying disease and by the extent of mitigation and social distancing measures imposed. This model allows for quantitative statements regarding the tradeoff between the severity and timing of suppression of the disease through social distancing and the progression of the disease in the population. The particular model studied is from Wang et al. (2020). ${ }^{4}$ Example applications of the model are provided. Special attention is given to the question of if and when the fraction of active infections in the

\footnotetext{
${ }^{1}$ See Ferguson et al. (2020) for a most recent model-based discussion of the public health implications of alternative social distancing and mitigation measures. The scenarios outlined in this paper are sobering. https://www.imperial.ac.uk/media/imperial-college/medicine/sph/ide/ gida-fellowships/Imperial-College-COVID19-NPI-modelling-16-03-2020.pdf

${ }^{2}$ Several early analyses of the economic impact of COVID-19 are coming out. Gourinchas (2020) and McKibbin and Roshen (2020) are useful discussions of the tradeoff between public health and economic impact. Barro et al. (2020) uses data from the 1918-19 Spanish Flu epidemic to put bounds on the impacts of COVID-19 on mortality and economic output.

${ }^{3}$ Here I consider a model in which agents cannot get the disease again once they have transitioned into the $R$ state. It is not yet clear whether this assumption is correct for COVID-19.

${ }^{4}$ https://doi.org/10.1038/s41421-020-0148-0
} 
population exceeds $1 \%$ (at which point the health system is forecast to be severely challenged) and 10\% (which may result in severe staffing shortages for key financial and economic infrastructure) as well as the cumulative burden of the disease over an 18 month horizon. ${ }^{5}$

Anderson et al. (2020) published online in The Lancet discusses the issues considered in this note. ${ }^{6}$ The figure 1 , taken from that paper illustrates qualitatively the impact of mitigation measures on the cumulative and peak incidence of the disease over a time horizon of 12 months together with the concern that relaxation of social distancing measures too early would lead to a resurgence of the disease. The aim of this note is to introduce economists to the quantitative implications of such models for alternative mitigation efforts.

\footnotetext{
${ }^{5}$ The scenarios considered here should not be considered definitive forecasts. They are intended only to allow the reader to see how a model of the progression of the epidemic might be applied to economic analysis of COVID-19 and to allow readers trained in economics to begin conversations with public health experts in this area.

${ }^{6}$ https://doi.org/10.1016/S0140-6736(20)30567-5
} 


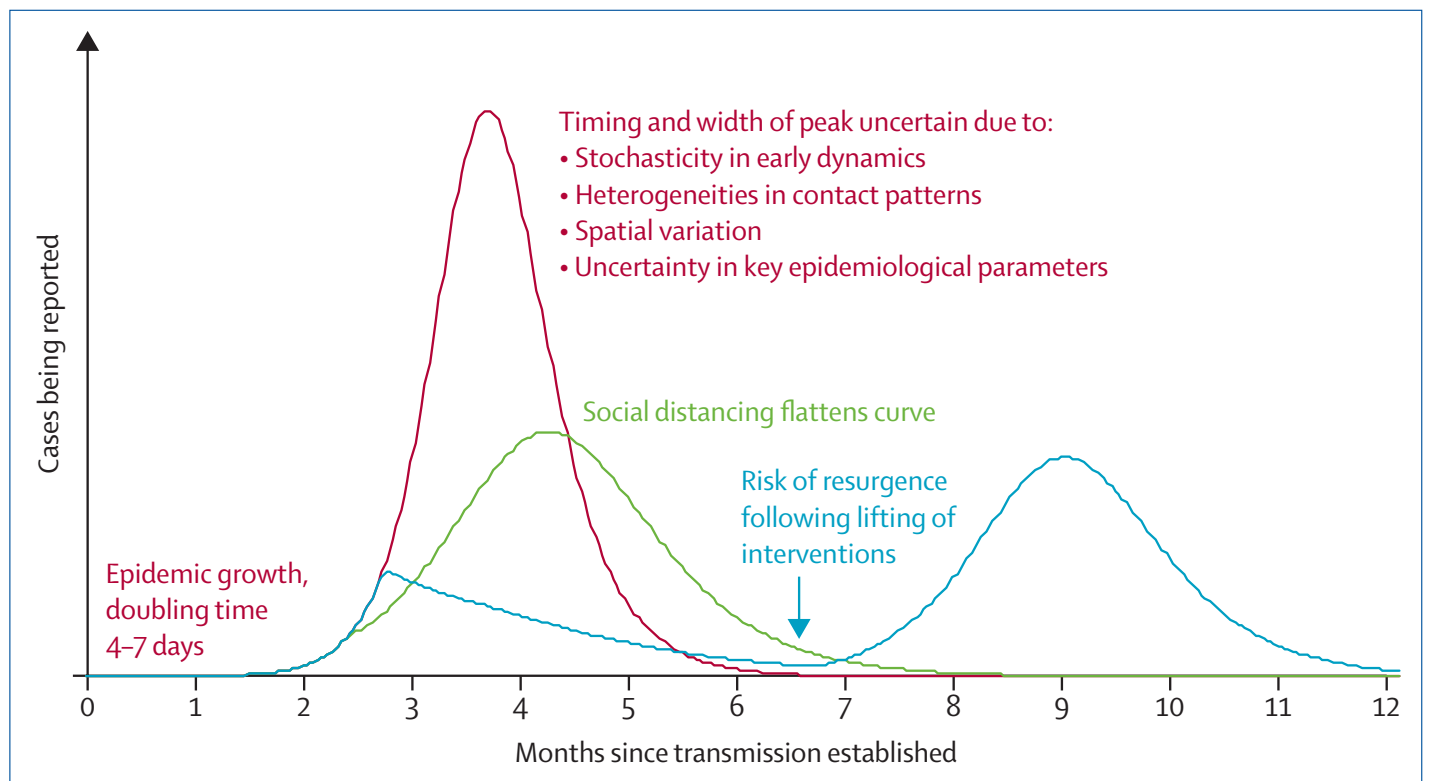

Figure: Illustrative simulations of a transmission model of COVID-19

A baseline simulation with case isolation only (red); a simulation with social distancing in place throughout the epidemic, flattening the curve (green), and a simulation with more effective social distancing in place for a limited period only, typically followed by a resurgent epidemic when social distancing is halted (blue). These are not quantitative predictions but robust qualitative illustrations for a range of model choices.

Figure 1: taken from Anderson et al. (2020) in The Lancet

\section{Main Conclusions:}

The main message for economists derived from simulations of this simple SIR model of the evolution of COVID-19 in the United States (and likely worldwide) is that it will likely require severe social distancing measures maintained for an entire year or even 18 months (until a vaccine can be developed) to avoid severe public health consequences.

Even putting aside concerns about public health, it appears that there is a significant economic tradeoff whether or not we impose social distancing - the economic costs of strong social distancing measures imposed for an entire 12-18 months on the one hand or the economic costs of a large cumulative burden of lost work time 
(and life) due to the disease. Which option would have the more severe economic consequences is hard to determine.

Even under severe social distancing scenarios, it is likely that the health system will be overwhelmed, which is indicated to happen when the portion of the U.S. population actively infected and suffering from the disease reaches 1\% (about 3.3 million current cases). ${ }^{7}$ More severe mitigation efforts do push the date at which this happens back from 6 months from now to 12 months from now or more, perhaps allowing time to invest heavily in the resources needed to care for the sick. It is clear that to avoid a health care catastrophe as is currently being experienced in Italy, prolonged severe social distancing measures will need to be combined with a massive investment in health care capacity.

Under almost all of the scenarios considered, at the peak of the disease progression, between $10 \%$ and $20 \%$ of the population (33 - 66 million people) suffers from an active infection at the same time. This level of infection in the population will likely require a significant diversion of the workforce from work to either self quarantine and recuperation or caring for these sick individuals for a period of weeks or more. It is likely that all of this would have to occur without adequate support from the health care system for those with dire cases of the disease even if we implement a large investment in healthcare. In the model simulations, this peak infection period occurs between 7-14 months from now. It is imperative to try to understand how critical healthcare, economic, and financial infrastructure would function in a period of such concentrated disease burden should this come to pass.

Many are making note of and taking heart from the apparent success of disease mitigation efforts in China, South Korea, Taiwan, Hong Kong, and Singapore. ${ }^{8}$ These

\footnotetext{
${ }^{7}$ See "What does the coronavirus mean for the U.S. health care system? Some simple math offers alarming answers" available here https://www.statnews.com/2020/03/10/ simple-math-alarming-answers-covid-19/. See also this recent assessment from the European Centre for Disease Prevention and Control https://www.ecdc.europa.eu/sites/default/files/documents/ RRA-sixth-update-Outbreak-of-novel-coronavirus-disease-2019-COVID-19.pdf

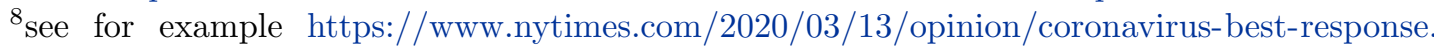
html. Wang et al. (2020) study the impact of mitigation efforts in Wuhan on the transmission of the epidemic. Hellewell et al. (2020) discuss the possibilities for containing the epidemic through
} 
areas have experienced remarkable success in reducing the number of new cases and slowing the growth of the cumulative number of cases from an exponential to a linear path through a variety of mitigation measures. But is it safe for these countries to begin to relax their mitigation efforts in the near future so as to resume economic activity? Or, as discussed in Anderson et al. (2020), would the disease simply re-emerge?

To answer this question, I use the model to ask what happens if extremely severe mitigation efforts are imposed on a temporary basis (for a few months) and then gradually relaxed. The model predicts that once mitigation efforts are relaxed, the disease simply restarts its rapid progression and sweeps through the population in less than 18 months, reaching its peak infection rate about 450 days from now. Thus, while the mitigation success of these countries is good news, a much more sustained mitigation effort will be required to capitalize on this success.

In the remainder of this note, I present the model and simulation results.

\section{The Model:}

The model presented in this note is based on the model presented in Wang et al. (2020). ${ }^{9}$ A similar model applied to the Seattle area, presented in Klein et al. $(2020)^{10}$ was used in advising Seattle area public health officials on the public health measures undertaken there. Academic models presented to the CDC in February but not made public were discussed in the New York Times on March $13^{11}$. Various interactive web-based versions of these models are available online. ${ }^{12}$ This video provides an accessible introduction to the mathematics involved. ${ }^{13}$

\footnotetext{
isolation and contact tracing https://doi.org/10.1016/S2214-109X(20)30074-7

${ }^{9}$ available here https://www.nature.com/articles/s41421-020-0148-0

${ }^{10}$ available here https://institutefordiseasemodeling.github.io/COVID-public/

${ }^{11}$ see this article https://www.nytimes.com/2020/03/13/us/coronavirus-deaths-estimate.html

${ }^{12}$ See, for example https://neherlab.org/covid19/ and https://www.nytimes.com/interactive/ 2020/03/13/opinion/coronavirus-trump-response.html

${ }^{13}$ https://www.youtube.com/watch?v=Kas0tIxDvrg
} 
The model is as follows.

The population is set to $N$ which here we normalize to one, so all results should be interpreted in fractions of the relevant population.

At each moment of time, the population is divided into four categories that sum to the total (of one). These are susceptible (no immunity) $S$, exposed $E$, infected $I$, and recovered (or dead) $R$. These fractions of the population evolve over time as follows

$$
\begin{gathered}
d S / d t=-\beta_{t} \frac{S}{N} I \\
d E / d t=\beta_{t} \frac{S}{N} I-\sigma E \\
d I / d t=\sigma E-\gamma I \\
d R / d t=\gamma I \\
\beta_{t}=R_{t} \gamma
\end{gathered}
$$

Following Wang et al. (2020), the parameter $\gamma$ governing the rate (per day) at which infected people either recover or die is considered a fixed parameter of the disease and is set to $\gamma=1 / 18$ reflecting an estimated duration of illness of 18 days. Likewise, the parameter $\sigma$ governing the rate at which those exposed to the disease become infected is also considered a fixed parameter of the disease and is set to $\sigma=1 / 5.2$ reflected an estimated incubation period of the disease of 5.2 days.

The parameter $\beta_{t}$ is the rate at which individuals who are infected bump into other people and "shed" virus onto those people. Of the people that they bump into, fraction $S / N$ are susceptible and hence transition to being exposed. The parameter $R_{t}$ is the ratio of this meeting rate $\beta_{t}$ and the recovery + death rate $\gamma$ on day $t$. This parameter governing the ratio of the rates at which those susceptible become infected and those ill either recover or die varies over time and is controlled by social distancing and quarantine measures. We will experiment with various time paths for this rate $R_{t}$. 
We parameterize this rate $R_{t}$ to allow for an initial period of intense application of social distancing measures followed by a relaxation of these measures to allow economic activity to resume. We do so with the following parameters

$$
\begin{gathered}
R_{1 t}=R_{1,0} \exp \left(-\eta_{1} t\right)+\left(1-\exp \left(-\eta_{1} t\right) \bar{R}_{1}\right. \\
R_{2 t}=R_{2,0} \exp \left(-\eta_{2} t\right)+\left(1-\exp \left(-\eta_{2} t\right) \bar{R}_{2}\right. \\
R_{t}=\left(R_{1 t}+R_{2 t}\right) / 2
\end{gathered}
$$

Here $R_{0}=\left(R_{1,0}+R_{2,0}\right) / 2$ is the initial value of $R_{t}$ representing the spread of the disease in its initial phase.

The parameters $\bar{R}_{i}$ for $i=1,2$ indicate the long run values to which $R_{i t}$ converge. Thus, in the long run $R_{t}$ converges to $\left(\bar{R}_{1}+\bar{R}_{2}\right) / 2$. To get a U-shaped pattern for $R_{t}$, we make $R_{1 t}$ a rapidly declining function and $R_{2 t}$ a slowly rising function The parameter $\eta_{1}$ governs the rate at which $R_{1 t}$ falls towards $\bar{R}_{1}$. The parameter $\eta_{2}$ governs the rate at which $R_{2 t}$ rises towards $\bar{R}_{2}$.

This parameterization of $R_{t}$ adds three differential equations to our model

$$
\begin{gathered}
d R_{1 t} / d t=-\eta_{1}\left(R_{1 t}-\bar{R}_{1}\right) \\
d R_{2 t} / d t=-\eta_{2}\left(R_{2 t}-\bar{R}_{2}\right) \\
d R_{t} / d t=-\frac{1}{2} \eta_{1}\left(R_{1 t}-\bar{R}_{1}\right)-\frac{1}{2} \eta_{2}\left(R_{2 t}-\bar{R}_{2}\right)
\end{gathered}
$$

with initial conditions $R_{i, 0}$.

\subsection{Model Parameters:}

The parameters $\gamma$ and $\sigma$ are set following Wang et al. (2020) as discussed above.

The initial conditions for all experiments are set as follows. The initial value of 
$I$ is set to one in ten million, corresponding to 33 initial cases in the United States given a population of 330 million. The initial value of $E=4 I$, corresponding to an initial 132 individuals carrying the virus but not yet contagious. These values roughly correspond to early February of 2020 for the United States. There is a lot of uncertainty regarding the number of initial cases.

The value of $R_{0}$ corresponds to the transmission of the disease with no mitigation efforts. This is a critical parameter for evaluation the progression of the disease in the population and the economic costs of mitigation. Wang et al. (2020) consider a value of $R_{0}=3.1$. Remuzzi and Remuzzi (2020) ${ }^{14}$ estimate value of $R_{0}$ between 2.76 and 3.25 using data from the outbreak of the disease in Italy. This website reports an $R 0$ in China of 2.5. ${ }^{15}$ The model referenced in Appendix 3 of Anderson et al. (2020) is also $R_{0}=2.5$. The model in this article in the New York Times considers a value of $R_{0}=2.3{ }^{16}$ Zhang et al. $(2020)^{17}$ estimates a value of $R_{0}=2.28 \mathrm{using}$ data from the Princess Cruise ship. Fauci et al. (2020) ${ }^{18}$ report an $R_{0}$ of 2.2. The European Centre for Disease Control ${ }^{19}$ reports $R_{0}$ as ranging from 2-3.

\subsubsection{The impact of mitigation on transmission:}

Social distancing and other mitigation steps can reduce the transmission rate $R_{t}$. Wang et al. (2020) estimate a gradual reduction of $R_{t}$ in Wuhan from 3.1, to 2.6 then to 1.9, and finally to somewhere between 0.9 and 0.5 with increasingly severe mitigation efforts. Kucharski et al. $(2020)^{20}$ estimate that travel restrictions in Wuhan imposed on January 23rd reduced $R_{t}$ from 2.35 to close to 1 one week later. Li et al. (2020) estimate that mitigation efforts in Wuhan reduced $R_{t}$ from an

\footnotetext{
${ }^{14}$ https://doi.org/10.1016/S0140-6736(20)30627-9

${ }^{15}$ https://www.isglobal.org/en/coronavirus-lecciones-y-recomendaciones

${ }^{16}$ https://www.nytimes.com/interactive/2020/03/13/opinion/coronavirus-trump-response.html

${ }^{17}$ https://www.ncbi.nlm.nih.gov/pubmed/32097725

${ }^{18}$ https://www.nejm.org/doi/full/10.1056/NEJMe2002387

${ }^{19}$ https://www.ecdc.europa.eu/sites/default/files/documents/RRA-sixth-update-Outbreak-of-novel-coronavirus-dise pdf

${ }^{20}$ https://doi.org/10.1016/S1473-3099(20)30144-4
} 
initial value of 2.38 to an average of 1.36 during the period January 24 to February 3 and an average of 0.99 during the period January 24 to February 8. I take these types of estimates as a guideline in parameterizing a scenario with temporary but severe mitigation measures imposed. I consider a reduction in transmission from 3.0 to 1.6 as more in line with milder social distancing measures. ${ }^{21}$ Anderson et al. (2020) discuss the uncertainties surrounding estimating the effect of mitigation. It is critical to revisit the effectiveness of mitigation efforts in slowing the transmission of the disease as more information comes in from current efforts underway.

I describe the mitigation scenarios I consider below.

\section{Model Experiment 1: Constant Mitigation over 18 months}

In my first computational experiment with the model, I ask what happens over the next 18 months holding fixed disease characteristics and mitigation efforts for the entire time period? To answer this question, I simulate the model under various fixed assumptions regarding the parameter $R_{t}=R_{0}$ governing the effectiveness of social distancing efforts of increasing severity in slowing the spread of the disease. ${ }^{22}$

I consider constant values of $R_{t}=R_{0}$ of 1.6, 1.8, 2.0,2.2, 2.5, 2.8 and 3.0. representing different levels of disease transmission under different mitigation scenarios. ${ }^{23}$ Note that if the true $R_{0}$ for COVID-19 in the US is 3 , then $R_{t}=1.6$ represents something close to a $50 \%$ reduction of the transmission rate through mitigation measures.

I plot the results for the cumulative cases as a fraction of the population over the

\footnotetext{
${ }^{21}$ This website https://neherlab.org/covid19/ considers "weak" mitigation as capable of reducing $R_{t}$ to $80 \%$ of $R_{0}$, "moderate" mitigation as reducing $R_{t}$ to $60 \%$ of $R_{0}$, and "strong mitigation" as reducing $R_{t}$ to $40-45 \%$ of $R_{0}$.

${ }^{22}$ To solve the system of differential equations, I use Matlab's ode113 solver and I set the the options to opts = odeset('Reltol',1e-13,'AbsTol',1e-14,'Stats','on')

${ }^{23}$ To produce a constant value of $R_{t}$ in the model, I set $\bar{R}_{1}=\bar{R}_{2}=R_{1,0}=R_{2,0}=R_{0}$. The values of $\eta_{1}$ and $\eta_{2}$ are arbitrary in this case and can be set to 1 .
} 
course of 18 months under each scenario for the transmission rate $R_{t}=R_{0}$ in Figure 2. Note that under almost all scenarios considered, more than $2 / 3$ of the population contracts the disease over the course of 18 months, but it takes 200 days for the cumulative number of cases to build to a crescendo, ripping through the population between 150 and 400 days from now, suggesting a substantial delay in the full impact of this disease. It is only in the case that $R_{t}=1.6$ that the disease is contained to a small fraction of the population over the time period until which time a vaccine might become available.

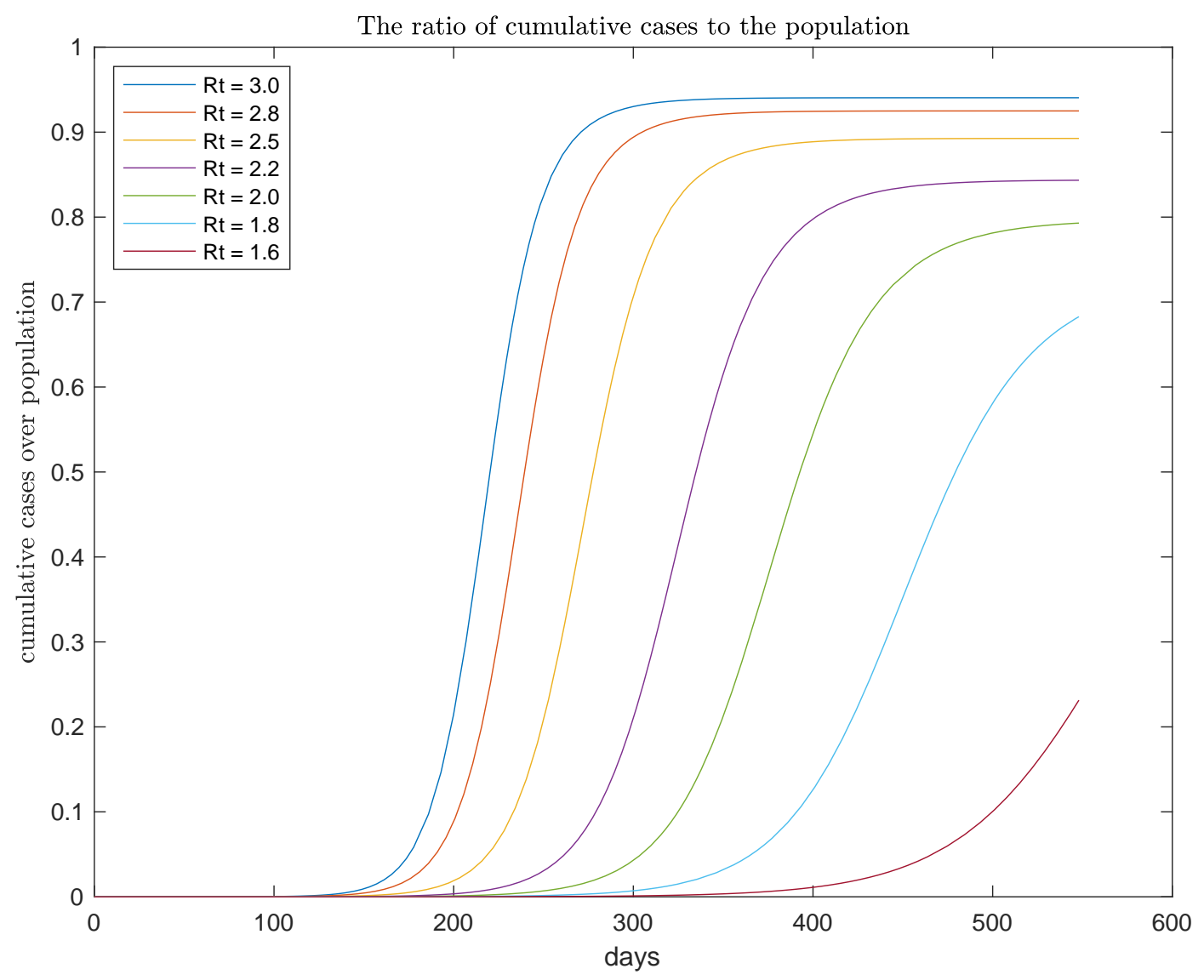

Figure 2: Cumulative Cases as a fraction of the population over 18 months under different values of $R_{t}=R_{0}$ held constant over the entire 18 month time period 
I plot the results for the fraction of the population with an active infection over the course of 18 months under each scenario for the transmission rate $R_{t}=R_{0}$ in Figure 3. As discussed above, a rough estimate is that the health system is overwhelmed when this fraction exceeds $1 \%$. The peak incidence in each case is substantially above this threshold. It appears to take 150-200 days to reach this threshold, with peak incidence coming in 250-475 days. It is clear that reductions in $R_{t}$ through mitigation efforts do "flatten the curve", but perhaps not by as much as might be hoped unless $R_{t}$ can be held to 1.6 or below for the entire 18 month time period.

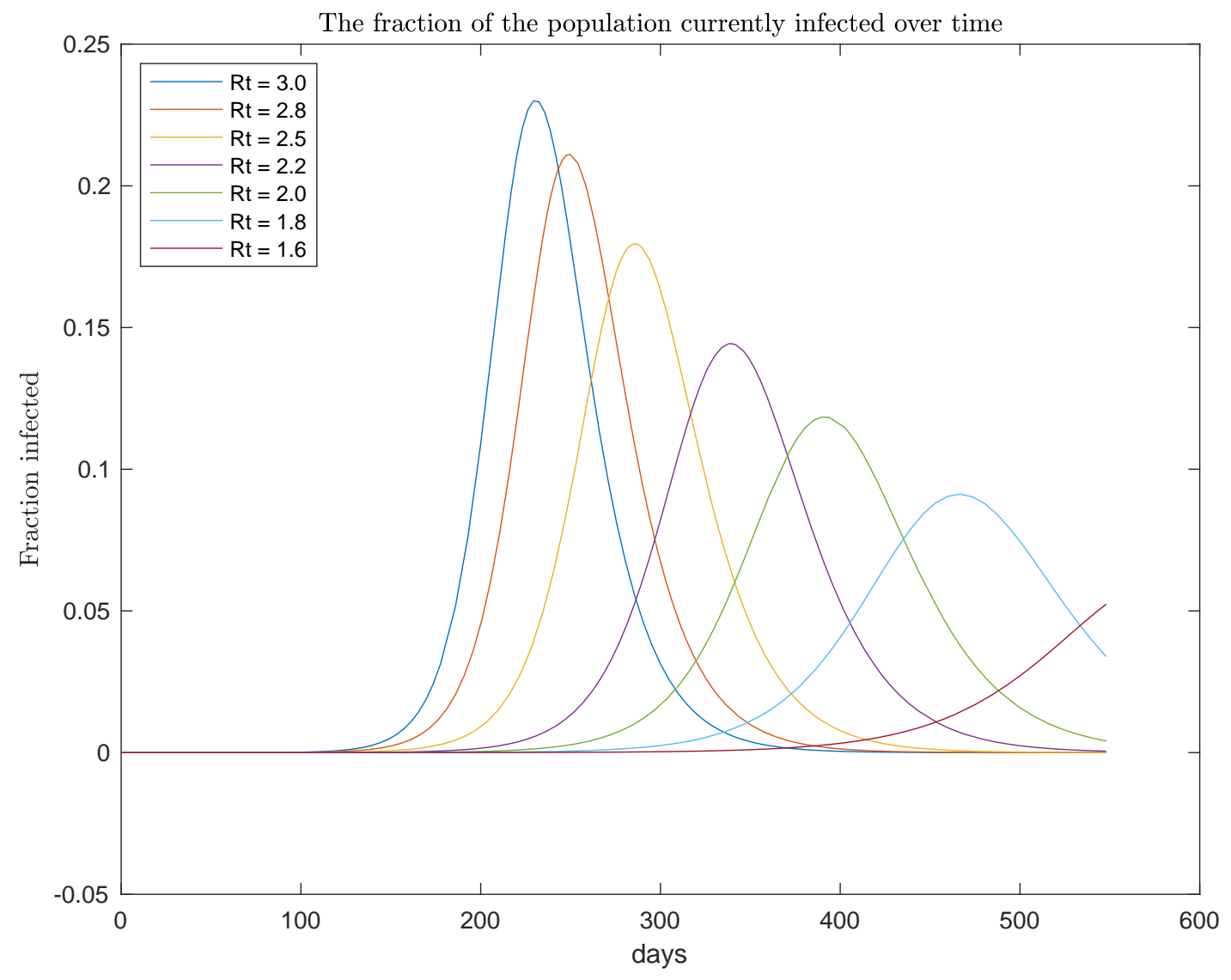

Figure 3: Fraction of the population with an active infection over 18 months under different values of $R_{t}=R_{0}$ held constant over the entire 18 month time period 
Finally, I examine the extent to which early information about the epidemic is helpful in distinguishing which scenario is most likely. To do so, I zoom in on the number of cumulative cases recorded in the early states of the epidemic over the first two months or so in figure 4. It appears that it might be difficult to distinguish which of these scenarios is the relevant one given the uncertainties in actually measuring the extent of the epidemic in its early phases. ${ }^{24} 25$

\footnotetext{
${ }^{24}$ Note that the doubling time for the different values of $R_{t}$ implied by the early phase of this experiment are $4.9,5.9,5.7,7.3,8,6,9.3$, and 12.0 days respectively going from the largest to smallest value of $R_{0}$ considered.

${ }^{25} \mathrm{See} \mathrm{Li}$ et al. (2020) for a discussion of the extent of unmeasured cases of coronavirus. https: //science.sciencemag.org/content/early/2020/03/13/science.abb3221.full
} 


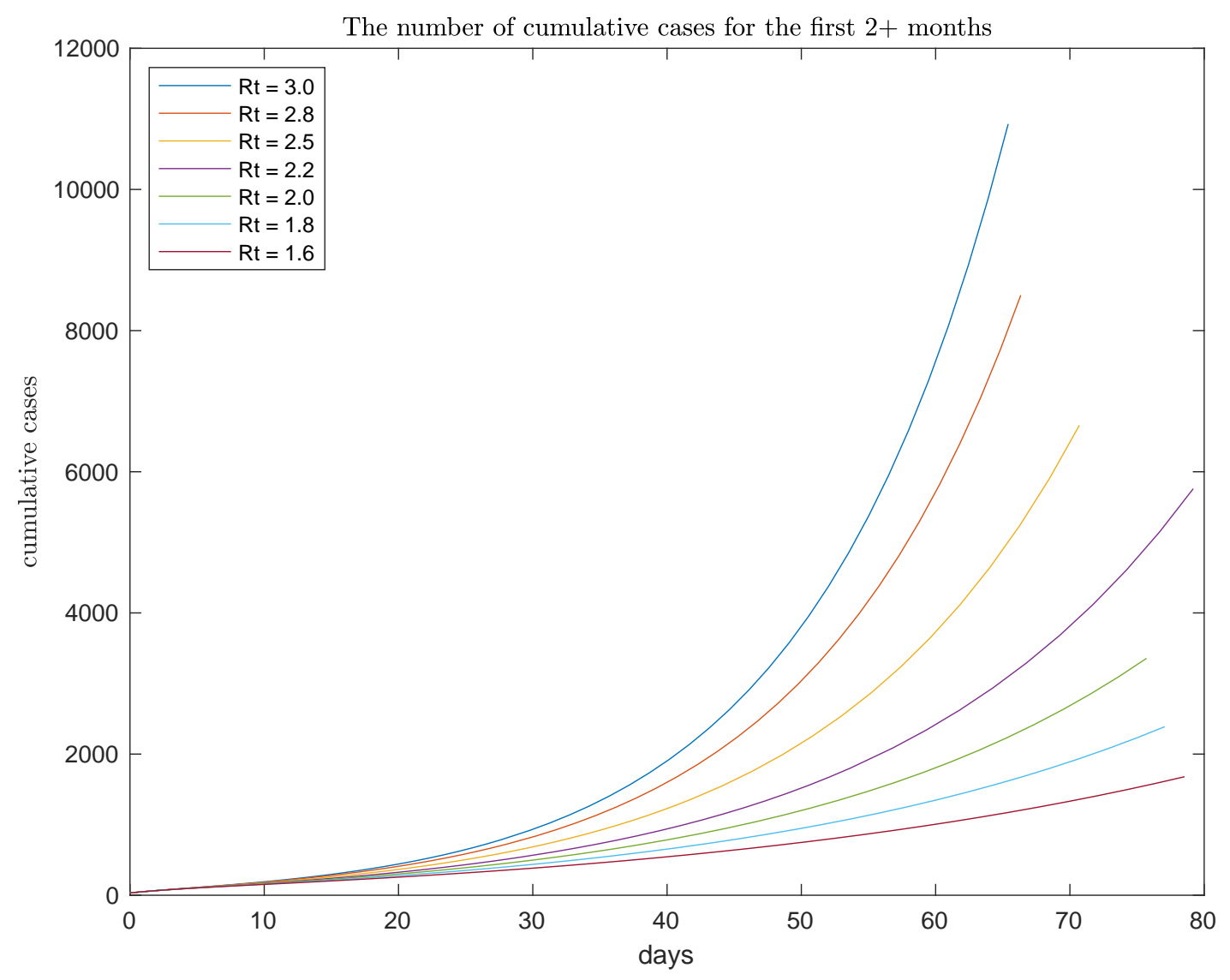

Figure 4: Cumulative Cases as a fraction of the population over 2+ months under different values of $R_{t}=R_{0}$ held constant over the entire time period

\section{Model Experiment 2: Speed of Mitigation}

In this second model experiment, I consider the impact of changing the speed of mitigation. In all the scenarios considered, I set $R_{0}=3.0$ and set $R_{\infty}=1.6$. I consider alternative speeds with which $R_{t}$ falls from this initial value to this long-run 
value (under permanently maintained mitigation measures). ${ }^{26}$

In Figure 5, I plot the various speeds with which reductions in $R_{t}$ are implemented over an 18 month period. In Figures 6 and 7 I plot the corresponding paths for cumulative cases and actively infected as a fraction of the population over the 18 months. The results of this simulation show remarkably small benefits to speedy application of mitigation measures in terms of reducing the peak fraction of the population infected. The primary benefit of speedy mitigation appears to be in delaying that period of peak infection. Further examination of this point is required.

\footnotetext{
${ }^{26}$ To implement this experiment, I set $\bar{R}_{1}=\bar{R}_{2}=1.6$ and $\eta_{1}=\eta_{2}$ equal to values of $1 / 5,1 / 10,1 / 20,1 / 50,1 / 100$ corresponding to very fast, fast, moderate, slow, and very slow scenarios repectively.
} 


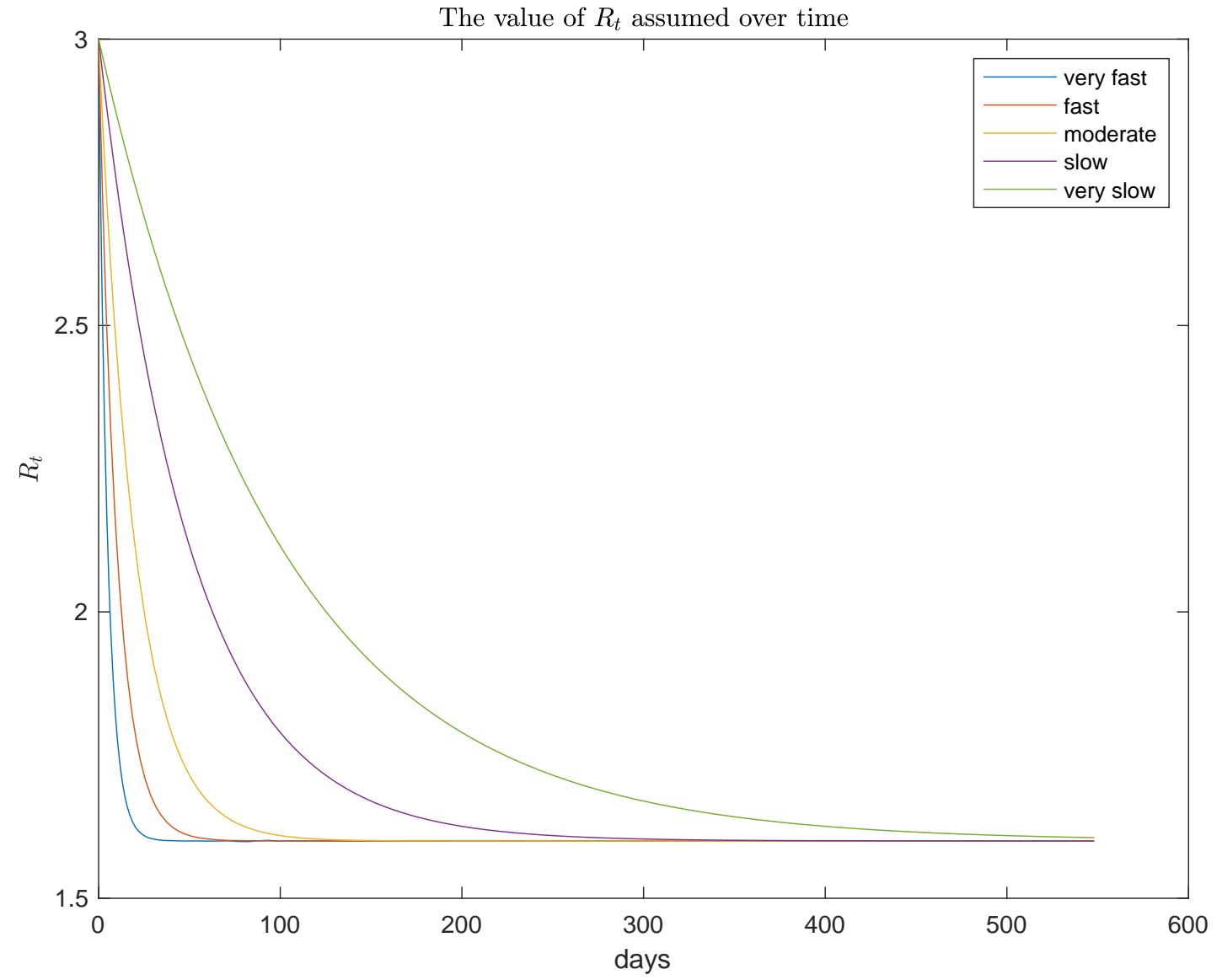

Figure 5: Reduction in $R_{t}$ through mitigation imposed at various speeds 


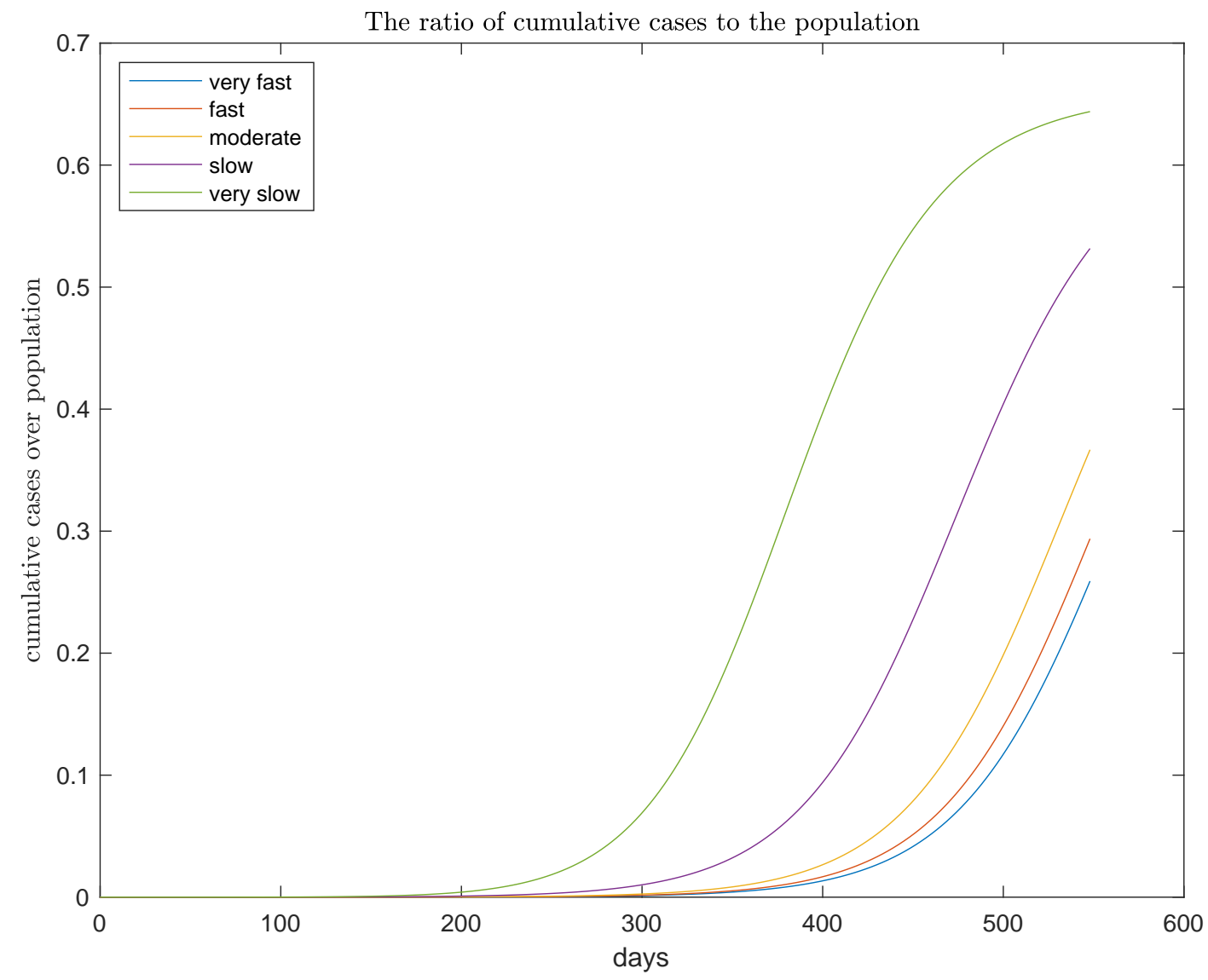

Figure 6: Cumulative cases as a fraction of the population under mitigation imposed at various speeds 


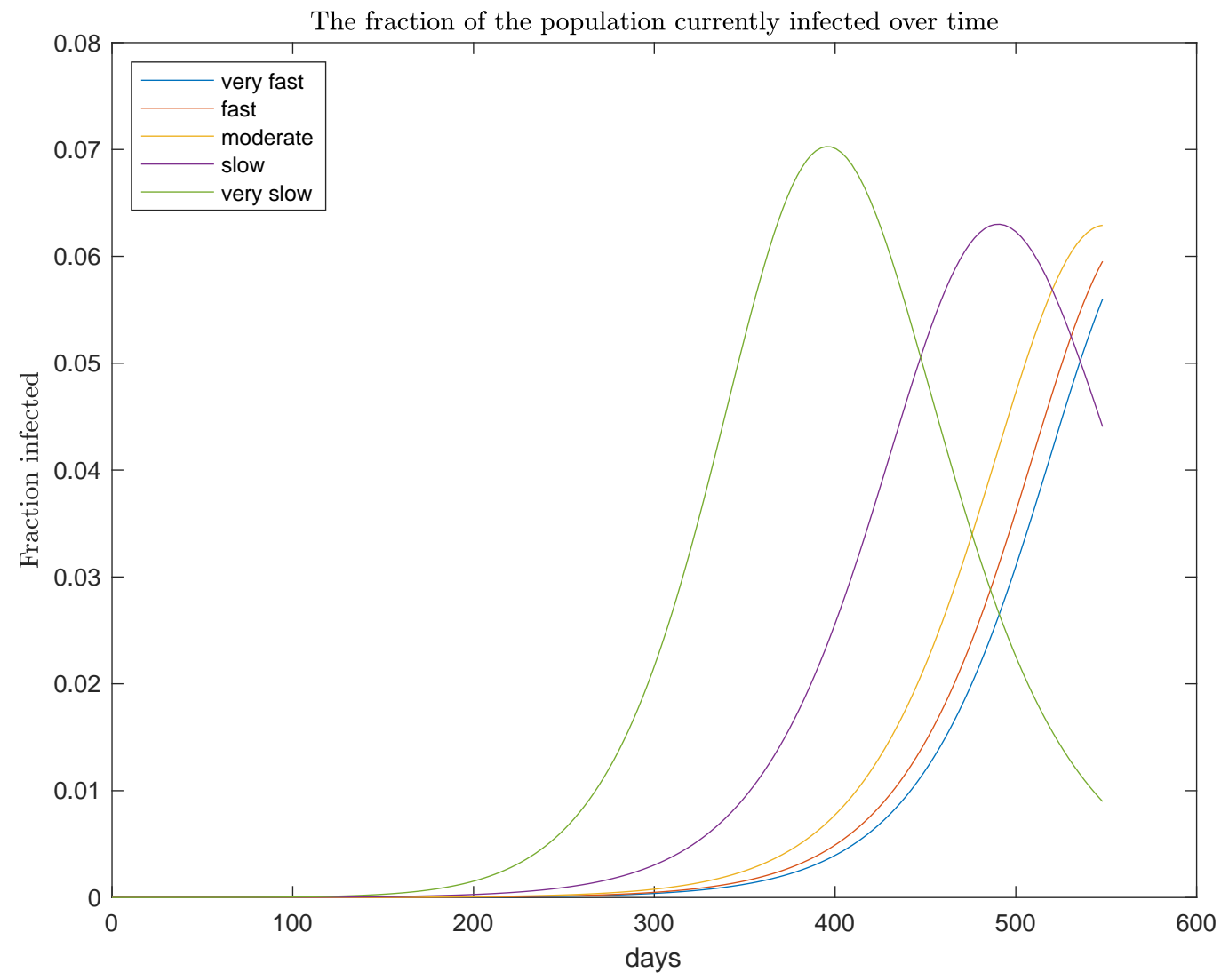

Figure 7: Active infections as a fraction of the population under mitigation imposed at various speeds

\section{Model Experiment 3: Temporary imposition of extremely severe mitigation measures}

In this third model experiment, I consider a path for mitigation efforts that results initially in a very sharp reduction in $R_{t}$ and then a gradual relaxation of these mitigation of these efforts.

The path of $R_{t}$ considered is shown in Figure 8 . Note that here I assume that 
these efforts result in values of $R_{t}$ well below 1 for a few months, which results in a shrinkage of the epidemic during those months. ${ }^{27}$

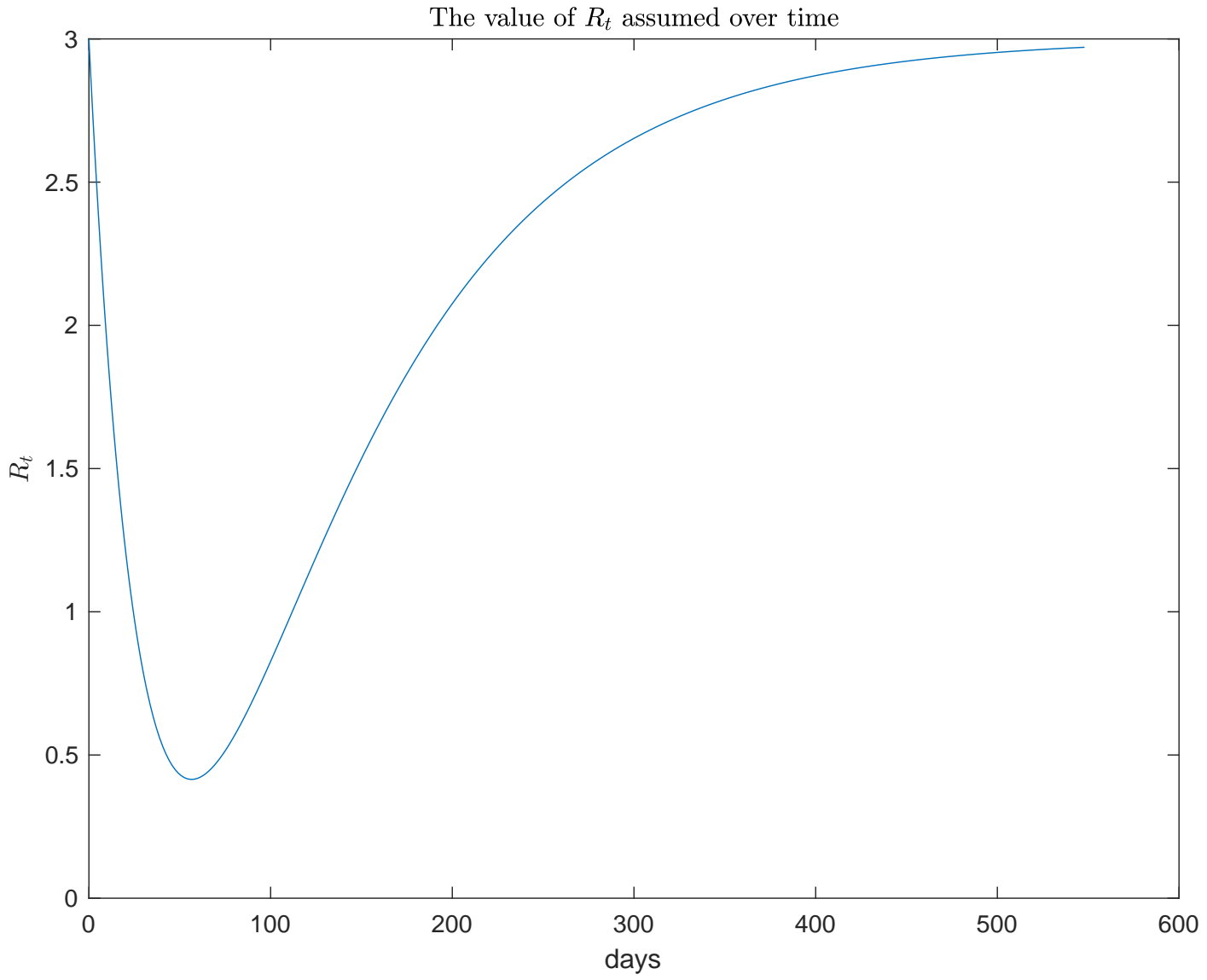

Figure 8: Path for $R_{t}$ under temporary extreme mitigation efforts

In Figures 9 and 10, I show the evolution of the cumulative cases of this epidemic and the number of people actively infected in the first several months under this temporary severe mitigation. As shown in these figures, the path for $R_{t}$ assumed does result in a "bending of the curve" from exponential to less than linear growth and a

\footnotetext{
${ }^{27}$ The parameter values that generate this path are $R_{1,0}=10, R_{2,0}=-4, \bar{R}_{1}=-4, \bar{R}_{2}=10$, and $\eta_{1}=1 / 35, \eta_{2}=1 / 100$
} 
substantial reduction in the number of people actively infected just as seen recently in China, South Korea, Taiwan, Singapore, and Hong Kong. Is this temporary success grounds for optimism?

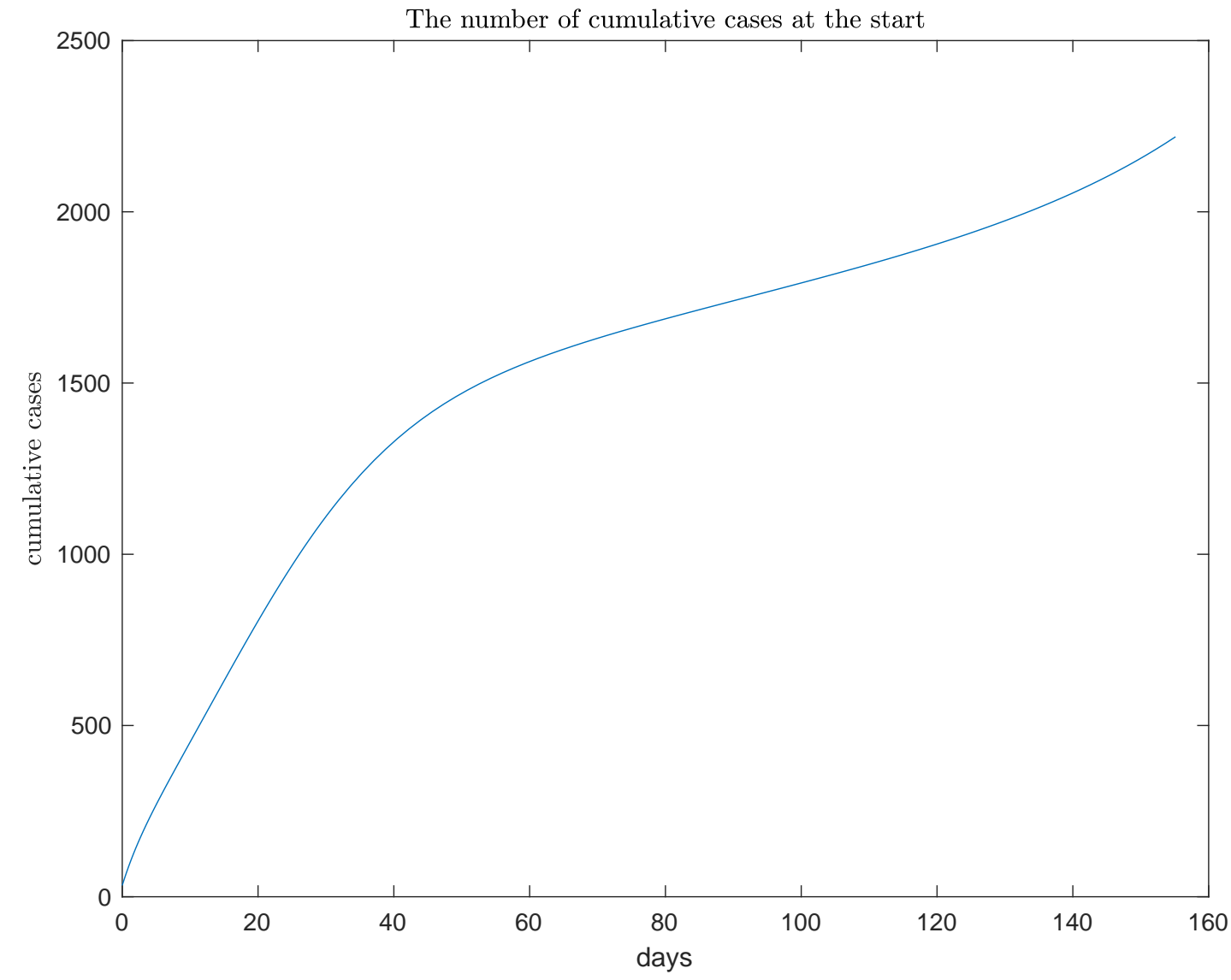

Figure 9: Cumulative cases as a fraction of the population under temporary severe mitigation efforts 


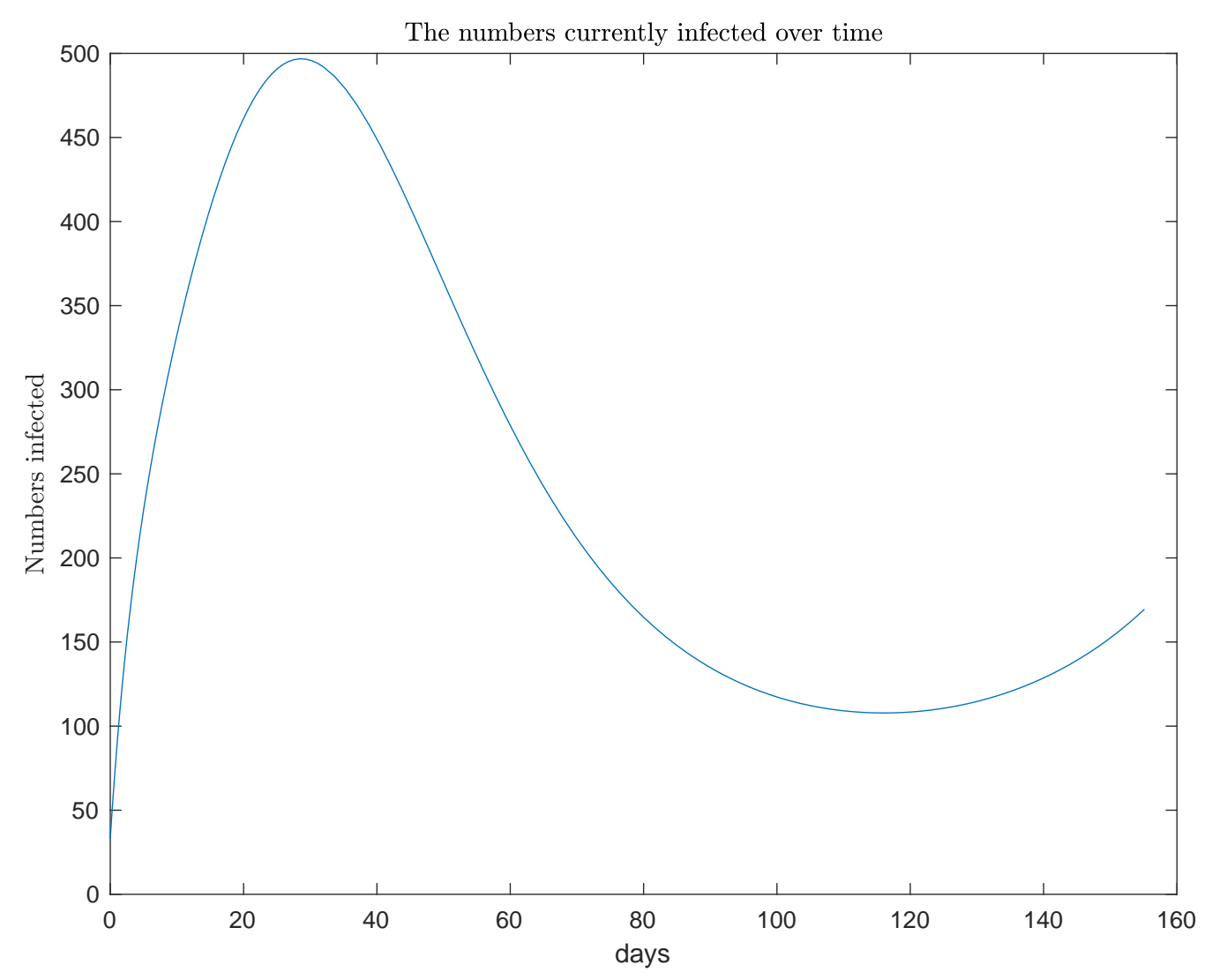

Figure 10: Number of people actively infected under temporary severe mitigation efforts

In figures 11 and 12, I show the path for cumulative cases and those actively infected as a fraction of the total population over the full 18 month period that includes relaxation of the severe mitigation. As is clear from the figures, the epidemic comes roaring back early in its second year if mitigation is relaxed. 


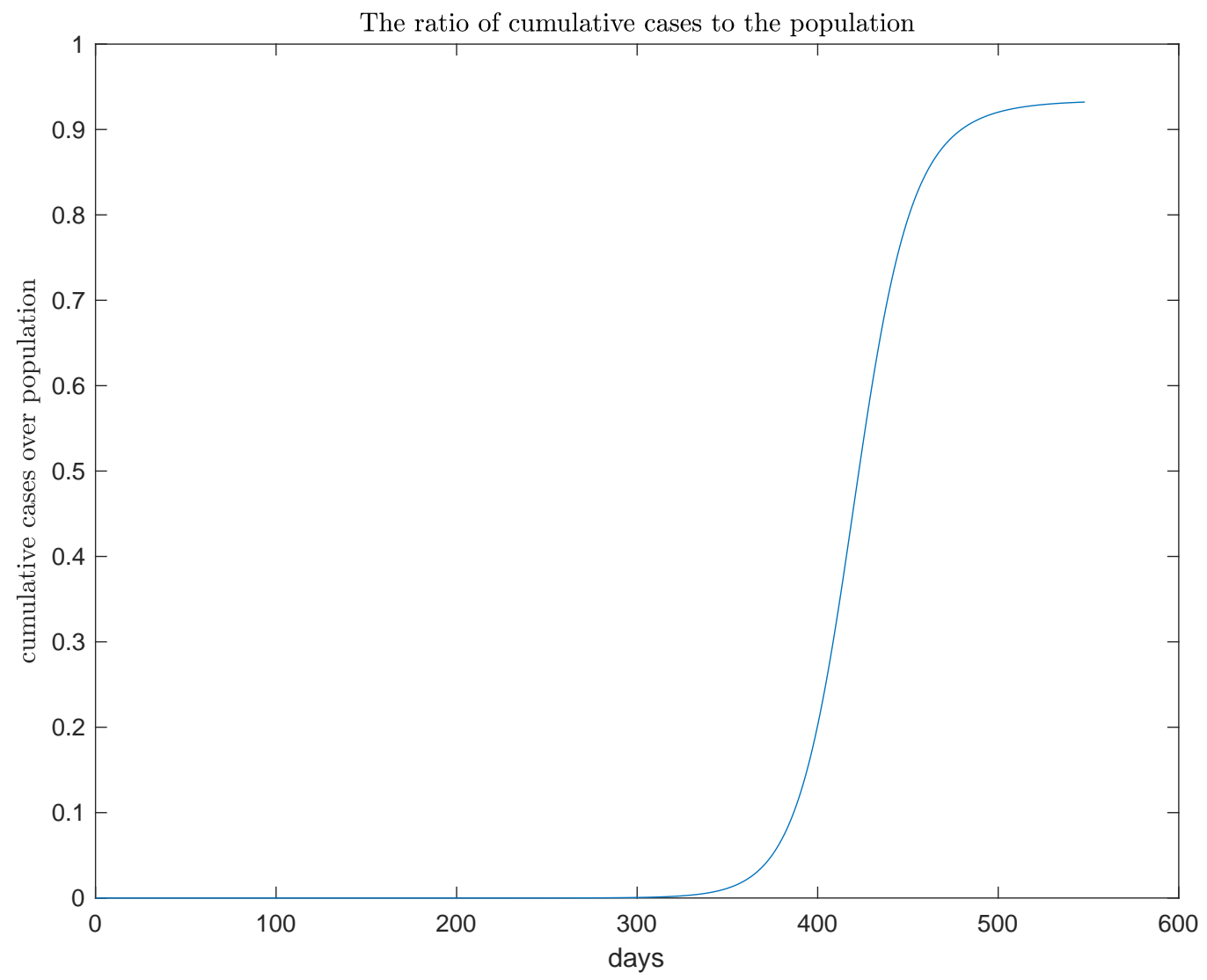

Figure 11: Cumulative cases as a fraction of the population under under temporary severe mitigation efforts 


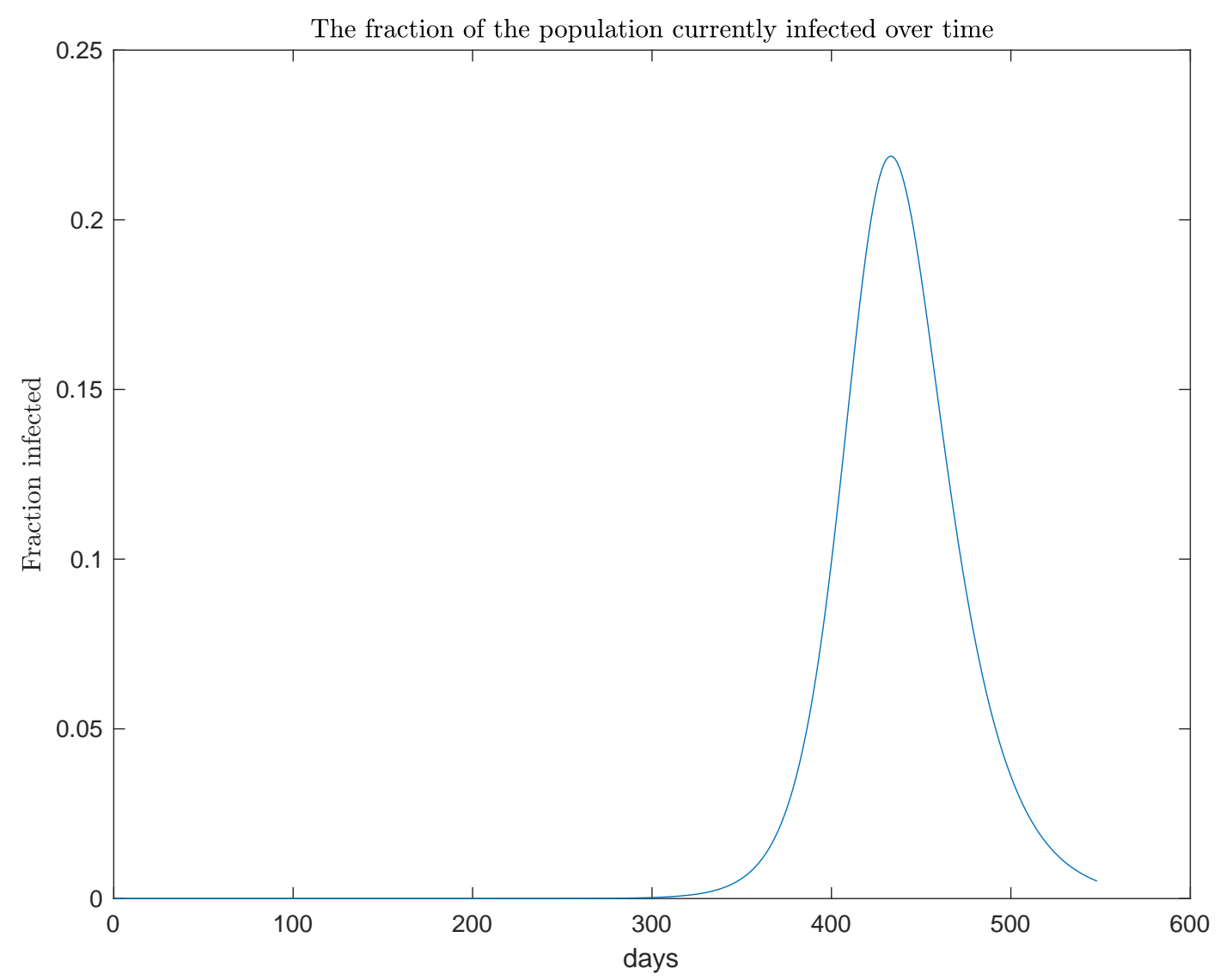

Figure 12: Active infections as a fraction of the population undertemporary severe mitigation efforts

\section{Conclusion}

The simulations of the model in this note, and, even more so, the fully detailed analysis in Ferguson et al. (2020), paint a grim picture of the choices regarding public health that policymakers face in mitigating the impact of the COVID-19 pandemic. What is urgently needed is an economic analysis of the economic consequences of the mitigation steps currently being implemented and contemplated going forward so that economic tradeoffs between public health and the economy can be considered 
quantitatively. I hope that academic and policy economists find this model useful in carrying out that analysis. 


\section{References}

Roy M. Anderson, Hans Heersterbeek, Don Klinkenberg, and T. Dierdre Hollingsworth. How will country-based mitigation measures influence the course of the covid-19 epidemic? The Lancet, March 2020.

Robert Barro, Jose F. Ursua, and Joanna Weng. The coronavirus and the great influenza epidemic: Lessons from the "spanish flu" for the coronavirus's potential effects on mortality and economic activity. March 2020.

Anthony S. Fauci, H' Clifford Lane, and Robert R. Redfield. Covid-19 — navigating the uncharted. New England Journal of Medicine, February 2020.

Neil M Ferguson, Daniel Laydon, Gemma Nedjati-Gilani, Natsuko Imai, Kylie Ainslie, Marc Baguelin, Sangeeta Bhatia, Adhiratha Boonyasiri, Zulma Cucunubá, Gina Cuomo-Dannenburg, Amy Dighe, Ilaria Dorigatti, Han Fu, Katy Gaythorpe, Will Green, Arran Hamlet, Wes Hinsley, Lucy C Okell, Sabine van Elsland, Hayley Thompson, Robert Verity, Haowei Volz, Erik Wang, Yuanrong Wang, Patrick GT Walker, Caroline Walters, Peter Winskill, Charles Whittaker, Christl A Donnelly, Steven Riley, and Azra C Ghani. Impact of non-pharmaceutical interventions (npis) to reduce covid19 mortality and healthcare demand. Technical report, Imperial College COVID-19 Response Team, March 2020.

Pierre Olivier Gourinchas. Flattening the pandemic and recession curves. https://drive.google.com/file/d/1mwMDiPQK88x27JznMkWzEQpUVm8Vb4WI/ view?usp=sharing, March 2020.

Joel Hellewell, Sam Abbott, Amy Gimma, Nikos Bosse, Christopher Jarvis, Timothy Russell, James D. Munday, Adam J Kurcharski, and W John Edmunds. Feasibility of controlling covid-19 outbreaks by isolation of cases and contacts. The Lancet, February 2020. 
Dan Klein, Brittany Hagerdorn, Cliff Kerr, Hu Hao, Trevor Bedford, and Mike Famulare. Working paper - model-based estimates of covid-19 burden in king and snohomish counties through april 7, 2020. March 2020.

Adam J. Kucharski, Timothy W Russell, Charlie Diamond, Yang Liu, John Edmunds, and Sebastian Funk. Early dynamics of transmission and control of covid-19: a mathematical modelling study. The Lancet, March 2020.

Ruiyun Li, Sen Pei, Bin Chen, Yimeng Song, Tao Zhang, Wan Yang, and Jeffrey Shaman. Substantial undocumented infection facilitates the rapid dissemination of novel coronavirus (sars-cov2). Science, March 2020.

Warren McKibbin and Fernando Roshen. The global macroeconomic impacts of covid-19: Seven scenarios. https://www.brookings.edu/research/ the-global-macroeconomic-impacts-of-covid-19-seven-scenarios/, March 2020.

Andrea Remuzzi and Giuseppe Remuzzi. Covid-19 and italy: what next? The Lancet, March 2020.

Huwen Wang, Zezhou Wang, Yinqiao Dong, Ruijie Chang, Chen Xu, Xiaoyue Yu, Shuxian Zhang, Lhakpa Tsamlag, Meili Shang, Jinyan Huang, Ying Wang, Gang Xu, Tian Shen, Xinxin Zhang, and Yong Cai. Phase-adjusted estimation of the number of coronavirus disease 2019 cases in wuhan, china. Cell Discovery, 6(1):10, 2020.

Sheng Zhang, MengYuan Diao, Wenbo Yu, Lei Pei, Zhaofen Lin, and Dechang Chen. Estimation of the reproductive number of novel coronavirus (covid-19) and the probable outbreak size on the diamond princess cruise ship: A data-driven analysis. International Journal of Infections Diseases, February 2020. 